\title{
Polimorfismos G691S, L769L y S836S del proto-oncogen RET no se asocian a mayor riesgo de cáncer medular tiroideo esporádico en pacientes chilenos
}

\author{
Nelson Wohllk $G^{1,2}$, Emiliano Soto $C^{3}$, Maritza Bravo $A^{2 a}$, \\ Pedro Becker $\mathrm{C}^{2,4}$. \\ G691S, L769L and S836S RET proto- \\ oncogene polymorphisms are not \\ associated with higher risk to sporadic \\ medullary thyroid carcinoma in Chilean \\ patients
}

Background: Medullary thyroid carcinoma (MTC) may occur either as sporadic or as hereditary. Even though the sporadic form corresponds to the majority of cases, the pathogenesis is still unclear. Several polymorphisms of the RET proto-oncogene, including those located in exon 11,13, 14 and 15 have been described in the general population and some of them seem to be overrepresented in sporadic MTC patients from European countries, especially G691S, L769L and S836S. Aim: To evaluate the allele frequencies of these variants in Chilean patients and controls and to determine if these polymorphisms would be associated with the development of sporadic MTC from a different genetic population base. Subjects and Methods: Fifty sporadic MTC patients and 50 normal subjects were tested for G691S, L769L, S836S and S904S polymorphisms. The extracted genomic DNA was initially analyzed by direct sequencing of PCR products in patients. The presence or absence of each polymorphism was also assessed in patients and in control by restriction digestion. Results: The allele frequencies showed a similar level of the G691S, L769L and S904S variants in both groups. Of interest, we found an underrepresentation of S836S polymorphism in the sporadic MTC group but this number was not statistically significant ( $p=0.141$ ). Conclusions: We did not find an over representation of the G691S, L769 and S836S. These results argue against the validity of the association of these polymorphisms as contributing factors in the development of sporadic MTC based on a Chilean population and raise questions about the importance of these polymorphisms overall (Rev Méd Chile 2005; 133: 397-402).

(Key Words: Proto-oncogene proteins; Protein-tyrosine kinase; Thyroid neoplasms)
Recibido el 13 de diciembre, 2004. Aceptado en versión corregida el 8 de marzo, 2005.
Trabajo financiado por Proyecto FONDECYT \#1980135.
${ }^{1}$ Sección Endocrinología Hospital del Salvador, Facultad de Medicina Universidad de Chile, Campus Oriente. ${ }^{2}$ Instituto de Estudios Médicos Avanzados. ${ }^{3}$ Facultad de Medicina, Universidad de Chile. ${ }^{4}$ Clínica Las Condes, Santiago Chile.
aTecnólogo Médico

Correspondencia a: Dr. Nelson Wohllk G. Rancagua 835,

Providencia. Fax: 251 8536. E mail: nwohllk@med.uchile.cl 
$\mathrm{E}^{1}$ cáncer medular del tiroides (CMT), comprende aproximadamente $5 \%$ a $10 \%$ de todas las neoplasias malignas del tiroides ${ }^{1,2}$. CMT es esporádico en $75 \%$ y $25 \%$ corresponde al síndrome hereditario autosómico dominante llamado neoplasias endocrinas múltiples (NEM) tipo 2A, 2B y cáncer medular familiar (Online Mendelian Inheritance in Man, MIM \#171400). El gen responsable de las NEM 2A, 2B y cáncer medular familiar es el proto-oncogen RET, localizado en el cromosoma 10q11.2, el cual codifica un receptor con actividad tirosina-quinasa ${ }^{3,4}$.

La variedad esporádica es definida ante la ausencia de historia personal o familiar sugerente de NEM 2 y ausencia de mutaciones de la línea germinal del proto-oncogen RET. Aunque la variedad esporádica es la forma de presentación más frecuente, los mecanismos responsables de su etiología y patogenia son prácticamente desconocidos ${ }^{5}$.

Por definición, las variantes genéticas de línea germinal, también llamadas polimorfismos, son variantes alélicas de un gen, las cuales no alteran la actividad funcional de la proteína codificada, independientemente de los cambios en la secuencia de la proteína. Una nueva estrategia para comprender un poco más la patogenia de estos tumores esporádicos, ha sido estudiar algunos polimorfismos que se ubican en los mismos exones en donde se han encontrado las mutaciones que dan cuenta de la mayoría de los CMT familiares, exceptuando el exón 10, el cual no presenta variante alélica. En pacientes europeos con CMT esporádico se ha descrito una mayor frecuencia de los polimorfismos ubicados en el exón 14 codón 836 (S836S, AGC >AGT), exón 11 codón 691 (G691S, GGT >AGT) y exón 13 codón 769 (L769L, CTT $>$ CTG) ${ }^{6-9}$. En lo que respecta al polimorfismo S836S las poblaciones estudiadas han sido alemana, estadounidense y española ${ }^{6,7}$. Los autores españoles señalan que este hallazgo podría ser más bien universal y no exclusivo de las poblaciones ya mencionadas ${ }^{7}$. Se ha sugerido que el polimorfismo S836S participaría en el proceso oncogénico, facilitando la expresión de ácido ribonucleico mensajero (ARNm) de RET, mecanismo también señalado para polimorfismos ubicados en otros genes ${ }^{10}$. Estudios recientes realizados en Italia, Francia y Polonia no han encontrado diferencias en las frecuencias alélicas de S836S y L769L en casos-controles ${ }^{8,9,12}$.
El primer objetivo de nuestro estudio fue evaluar la frecuencia alélica de los polimorfismos del proto-oncogen RET más frecuentemente estudiados, en una muestra de individuos chilenos y compararla con las series publicadas. El otro objetivo fue el determinar si las variantes G691S, L769L y S836S se asocian a un mayor riesgo de presentar CMT en una población genéticamente diferente a las europeas.

\section{PACIENTES, CONTROLES Y MÉTODO}

Pacientes. 50 pacientes no relacionados entre sí, portadores de CMT esporádico, procedentes de diferentes ciudades de Chile, fueron evaluados mediante estudio genético del proto-encogen RET, entre los años 1998 y 2001. La designación de esporádico se basó en la ausencia de historia familiar de CMT o de otras manifestaciones de NEM 2, ausencia de mutaciones de línea germinal en los exones 10,11, 13, 14, 15 y 16 del proto-oncogen RET como ha sido previamente reportado ${ }^{13}$. El estudio histológico revisado en todos los casos confirmó la presencia de un CMT unifocal y ausencia de hiperplasia de células C; la inmunohistoquímica del tumor fue positiva para calcitonina y negativa para tiroglobulina.

Controles. 50 individuos chilenos no relacionados entre sí, sin historia personal o familiar de cáncer de tiroides fueron considerados como grupo control. Tanto los pacientes como los controles eran étnicamente similares, con apellido materno y paterno predominantemente español y sin ancestro indígena en sus padres. El estudio fue aprobado por el comité de ética del Hospital del Salvador y se obtuvo consentimiento informado de los pacientes y controles.

Método. Muestra de ADN genómico: el ADN se aisló de una muestra de sangre periférica de acuerdo al método de Lahiri, usando el kit de DNAzol $\left.^{\circledR}{ }^{(G i b c o B R L}\right)^{14}$. Los exones 11, 13, $14 \mathrm{y}$ 15 y del proto-oncogen RET en donde se localizan los 4 polimorfismos analizados (G691S, L769L, S836S y S904S), fueron amplificados por reacción en cadena de polimerasa (PCR) en un volumen total de $40 \mu \mathrm{L}$, conteniendo $200 \mathrm{ng}$ de ADN genómico, $1 \mu \mathrm{mol} / \mathrm{l}$ de cada partidor cuyas secuencias aparecen en la Tabla 1; 1,5 mM/l de 
Tabla 1. Secuencia de los partidores para la amplificación por PCR de los exones 11, 13, 14 y 15 del proto-oncogen RET

\begin{tabular}{|cll|}
\hline Exón & Nombre & Secuencia de los partidores \\
\hline 11 & 11 IF & 5' GACACGGCAGGCTGGAGAGC 3' \\
& CRT19A & 5' CTTGAAGGCATCCACGGAGA 3' \\
13 & $13 F 2$ & 5' CTCTCTGTCTGAACTTGGGC 3' \\
& $13 \mathrm{IR}$ & 5' AGAACAGGGCTGTATGGAGC 3' \\
14 & $14 \mathrm{IF}$ & 5' AAGACCCAAGCTGCCTGAC 3' \\
& $14 \mathrm{IR}$ & 5' GCTGGGTGCAGAGCCATAT 3' \\
15 & $15 \mathrm{~F}$ & 5' GTGACCGCTGCCTGGCCATGG 3' \\
& $15 \mathrm{R}$ & 5' CCTAGGCTTCCCAAGGACTGCCTGC 3' \\
\hline
\end{tabular}

MgCl2, $10 \mathrm{mM} / \mathrm{l} \mathrm{Tris-HCl} \mathrm{(pH} \mathrm{9),} 50 \mathrm{mM} / \mathrm{l} \mathrm{KCl,} 200$ $\mu \mathrm{M} / \mathrm{l}$ dNTPs y $1 \mathrm{U}$ Taq polimerasa (Promega). Las condiciones para la amplificación fueron diferentes para cada exón y ellas han sido descritas previamente ${ }^{15,16}$.

La secuenciación directa de los productos amplificados se hizo en los pacientes usando un método modificado del protocolo del thermosequenase radiolabeled terminator cycle sequencing $\mathrm{kit}^{\circledR}$ (USB Corporation) ${ }^{16}$. Un ejemplo de la secuencia del exón 13 en donde se ubica el polimorfismo L769L se muestra en la Figura 1. La presencia 0 ausencia de cada polimorfismo fue evaluada nuevamente en los pacientes y como único método en todos los controles mediante el análisis de restricción enzimática usando las enzi- mas: Ban I, Taq I, Alu I y Rsa I las cuales detectan los polimorfismos G691S, L769L, S836S y S904S, respectivamente. En breve: $10 \mu \mathrm{l}$ de la amplificación por PCR fue incubada según la temperatura sugerida por los fabricantes, con $2 \mathrm{U}$ de la enzima y su buffer respectivo en un volumen total de 20 $\mu l$. Los productos fueron posteriormente separados por electroforesis en gel de poliacrilmida al $10 \%$ y teñidos con bromuro de etidio para su visualización con luz ultravioleta. Se utilizaron muestras de pacientes como controles positivos y negativos para asegurar la actividad de las enzimas de restricción empleadas.

Estadística. El análisis estadístico se realizó con el test de Chi cuadrado $\left(\chi^{2}\right)$, considerándose significativo un valor $\mathrm{p} \leq 0,05$.

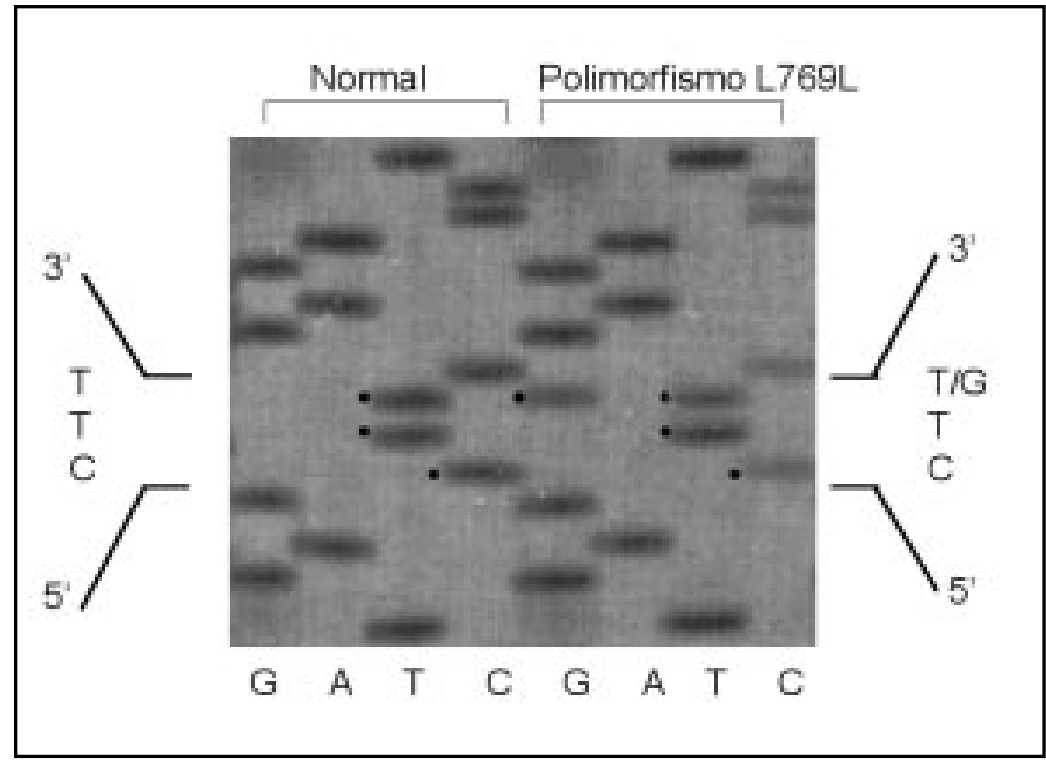

Figura 1. Secuencias parciales del exón 13 del proto-oncogen en 2 pacientes con CMT esporádico. Las letras inferiores corresponden a los nucleótidos Guanina (G), Adenina (A), Timina ( $\mathrm{T}$ ) y Citosina (C). Las secuencias se leen de abajo hacia arriba en la dirección $5^{\prime} \rightarrow$ 3'. Los nucleótidos del codón 769 aparecen destacados en círculos llenos. La parte izquierda de la Figura muestra la secuencia normal (CTT) y la de la derecha el polimorfismo L769L (CTT $\rightarrow$ CTG). 


\section{RESULTADOS}

Nuestras cifras muestran que las frecuencias alélicas de los polimorfismos G691S, L769L S904S son similares en nuestros pacientes y controles al igual que lo observado en pacientes de Alemania y Estados Unidos de Norte América (EE.UU) (Tabla 2). No encontramos una mayor frecuencia de las variantes G691S y L769L en los pacientes con CMT, como ha sido informado en pacientes italianos y polacos, respectivamente. Un hallazgo interesante fue el encontrar una menor frecuencia alélica del polimorfismo S836S en el grupo de pacientes (1/100 cromosomas, 1\%). Esta cifra no alcanzó una diferencia estadísticamente significativa con la cohorte control (CMT vs control, $\chi^{2}=2.171, p=0,141$ ). Como se puede observar también en la Tabla 2, este hallazgo es totalmente diferente a lo señalado por algunos estudios.

\section{DisCUSIÓN}

La etiología del CMT esporádico aún no ha sido elucidada. Mutaciones somáticas del proto-oncogen RET, predominantemente en el codón 918 (M916T), contribuyen al desarrollo de $25 \%$ a $50 \%$ de los casos reportados ${ }^{17-19}$. El primer estudio que llamó la atención acerca de la importancia de la variante S836S (AGC >AGT), informó una mayor frecuencia de esta variante, en el ADN genómico de pacientes con CMT esporádico provenientes de Alemania y EE.UU, al compararlos con controles de las mismas áreas geográficas $\left(9 \%\right.$ vs 3,6\%) ${ }^{6}$. En el mismo estudio, 8 de 9 pacientes que presentaban esta variante, también presentaban la mutación somática en el codón 918. Ellos sugirieron que la variante S836S estaría de algún modo relacionada con la mutación somática M918T y que podrían dar cuenta de la patogénesis de un subgrupo de pacientes con CMT. El mecanismo que ellos postulan es el que la variante

\section{Tabla 2. Frecuencia alélica ${ }^{1}$ de los polimorfismos en controles y pacientes chilenos con CM T esporádico y} de las publicadas en diferentes poblaciones europeas

\begin{tabular}{|c|c|c|c|c|c|c|c|c|c|c|c|c|}
\hline \multirow{5}{*}{ Polimorfismo } & \multicolumn{2}{|c|}{ Chile } & \multicolumn{2}{|c|}{$\begin{array}{c}\text { Alemania y } \\
\text { EE.UU }\end{array}$} & \multicolumn{2}{|c|}{ España $^{7}$} & \multicolumn{2}{|c|}{ Polonia $^{9}$} & \multicolumn{2}{|c|}{ Italia $^{8}$} & \multicolumn{2}{|c|}{ Francia $^{11}$} \\
\hline & C & CMT & $\mathrm{C}$ & CMT & C & CMT & C & CMT & C & CMT & $\mathrm{C}$ & CMT \\
\hline & $\mathrm{n}$ & $\mathrm{n}$ & $\mathrm{n}$ & $\mathrm{n}$ & $\mathrm{n}$ & $\mathrm{n}$ & $\mathrm{n}$ & $\mathrm{n}$ & $\mathrm{n}$ & $\mathrm{n}$ & $\mathrm{n}$ & $\mathrm{n}$ \\
\hline & $\%$ & $\%$ & $\%$ & $\%$ & $\%$ & $\%$ & $\%$ & $\%$ & $\%$ & $\%$ & $\%$ & $\%$ \\
\hline & \multicolumn{2}{|c|}{$\mathrm{p}$} & \multicolumn{2}{|c|}{$\mathrm{p}$} & \multicolumn{2}{|c|}{$\mathrm{p}$} & \multicolumn{2}{|c|}{$\mathrm{p}$} & \multicolumn{2}{|c|}{$\mathrm{p}$} & \multicolumn{2}{|c|}{$\mathrm{p}$} \\
\hline G691S & 100 & 100 & 140 & 94 & $\mathrm{n} / \mathrm{i}$ & $\mathrm{n} / \mathrm{i}$ & $\mathrm{n} / \mathrm{i}$ & $\mathrm{n} / \mathrm{i}$ & 212 & 212 & $\mathrm{n} / \mathrm{i}$ & $\mathrm{n} / \mathrm{i}$ \\
\hline \multirow[t]{2}{*}{$(\mathrm{AGT}>\underline{\mathrm{GGT}})$} & & 25 & 21 & 23 & & & & & 18,8 & 27,8 & & \\
\hline & \multicolumn{4}{|c|}{ NS $\quad$ NS } & & & \multicolumn{6}{|c|}{0,029} \\
\hline L769L & 100 & 100 & 140 & 96 & $\mathrm{n} / \mathrm{i}$ & $\mathrm{n} / \mathrm{i}$ & $\mathrm{n} / \mathrm{i}$ & 22 & 212 & 212 & 174 & 184 \\
\hline$(\mathrm{CTT}>\underline{\mathrm{CTG}})$ & 24 & 23 & 26 & 26 & & & & 36 & 24 & 21,6 & 26 & 22 \\
\hline & \multicolumn{2}{|c|}{ NS } & \multicolumn{2}{|c|}{ NS } & & & & & \multicolumn{2}{|c|}{ NS } & \multicolumn{2}{|c|}{ NS } \\
\hline S836S & 100 & 100 & 140 & 98 & 500 & 64 & $\mathrm{n} / \mathrm{i}$ & 82 & 212 & 212 & 174 & 184 \\
\hline \multirow[t]{2}{*}{$(\mathrm{AGC}>\mathrm{AGT})$} & 6 & 1 & 3,6 & 9 & 3,6 & 9,3 & & 1,2 & 8,4 & 6,1 & 5,2 & 6,5 \\
\hline & \multicolumn{2}{|c|}{ NS } & \multicolumn{2}{|c|}{0,01} & 0,043 & & & NS & & NS & & \\
\hline S904S & 100 & 100 & 140 & 94 & $\mathrm{n} / \mathrm{i}$ & $n / i$ & $\mathrm{n} / \mathrm{i}$ & $\mathrm{n} / \mathrm{i}$ & 212 & 212 & $\mathrm{n} / \mathrm{i}$ & $\mathrm{n} / \mathrm{i}$ \\
\hline \multirow[t]{2}{*}{$(\mathrm{TCC}>\mathrm{TCG})$} & 28 & 27 & 21 & 20 & & & & & 18,8 & 23,5 & & \\
\hline & \multicolumn{2}{|c|}{ NS } & \multicolumn{2}{|c|}{ NS } & & & & & \multicolumn{2}{|c|}{ NS } & & \\
\hline
\end{tabular}

${ }^{1}$ La frecuencia alélica fue calculada sobre el número de cromosomas analizados y es referida al alelo que presenta la mutación y cuya secuencia aparece entre paréntesis y subrayada.

Abreviaciones: C: Controles; CMT: Cáncer Medular Tiroides; N: número de cromosomas analizados; n/i: no informado. 
S836S causaría la creación o facilitación de un sitio para la eliminación de los intrones, lo cual conduciría a la formación de una proteína alterada. Esta alta frecuencia alélica fue confirmada en una serie de pacientes españoles, sugiriendo que este polimorfismo no estańa limitado exclusivamente a la población de Alemania y EE.UU, y que senía un hallazgo más bien universal ${ }^{7}$. Estudios recientes provenientes de Polonia, Francia e Italia no han encontrado mayor frecuencia de este polimorfismo en particular $8,9,11,12$. También se ha descrito últimamente una mayor frecuencia de los polimorfismos G691S y L769L El polimorfismo G691S es el único que determina una sustitución de aminoácido (glicina > serina) lo cual podría conferir a la proteína RET una estructura conformacional y electroquímicamente diferente $\mathrm{y}$, por lo tanto, una actividad funcional diferente. El estudio de la cohorte italiana aunque reportó una mayor frecuencia alélica de G691S en los pacientes, no pudo demostrar un aumento en la expresión del ARNm en tumores que presentaban el alelo mutado ${ }^{8}$. Otro hecho que hace pensar que este polimorfismo sería poco importante en la patogenia del CMT, es la alta frecuencia encontrada en sujetos sanos de todas las poblaciones estudiadas (Tabla 2), siendo difícil demostrar que estos sujetos tengan un mayor riesgo de desarrollar CMT. Respecto a la variante L769L, en pacientes polacos se encontró una mayor frecuencia en el subgrupo $\leq 30$ años al compararlos con los $>30$ años ( $36 \%$ vs $15 \%, p=0,04)^{9}$. Sin embargo, el número de pacientes estudiados era escaso y al incluir un grupo control no se encontraron diferencias en esta variante alélica ${ }^{12}$. Estudios en Francia tampoco han confirmado una mayor frecuencia de este polimorfismo (Tabla 2, ref. 11). Todos los estudios publicados hasta la fecha han considerado mayoritariamente poblaciones provenientes de Europa. No existe información en poblaciones de Centro y Sur Aménica. Estudio de polimorfismos en un grupo pequeño de pacientes brasileños (7 esporádicos y 4 NEM 2) y sin un grupo control para comparar, fue informado recientemente ${ }^{20}$.

\section{REFERENCIAS}

1. Rahue F, Frank-Raue K, Grauer A. Multiple Endocrine Neoplasia Type 2: Clinical Features and Screening. Endocr Metab Clin North Am 1994; 23: 137-56.
La población chilena actual es predominantemente mestiza y proviene de una mezcla étnica ancestral de colonizadores españoles y aborígenes locales (especialmente los grupos Araucanos Mapuches, Pehuenches y Huilliches), la cual ocurrió entre los siglos 16 y $18^{21,22}$.

Nuestro laboratorio, en el peńodo 1998-2001, se constituyó en el Centro Nacional de referencia para el estudio genético del proto-encogen RET en pacientes con CMT familiar, esporádico y sus familiares, por lo que nuestra cohorte representa la mayońa de los casos de CMT esporádicos diagnosticados antes y durante ese peńodo ${ }^{13}$. En esta cohorte de tamaño similar al de los estudios ya mencionados, no se encontraron diferencias en las frecuencias alélicas de los principales polimorfismos del proto-oncogen RET al estudiar el ADN genómico de los pacientes y controles. Estas diferencias podnían reflejar la variabilidad genética de nuestra población con respecto a la de otras poblaciones estudiadas (alemana, española, italiana, etc.), sin embargo resultados similares a los nuestros se han descrito en otras poblaciones de Europa, como se muestra en la Tabla 2. Estos resultados sugieren que las variantes alélicas estudiadas no tendrían un rol como factor de riesgo de CMT esporádico en la población chilena y especialmente en el caso del polimorfismo S836S, de la cual se ha dicho que podńa estar presente en todas la poblaciones estudiadas, nuestros hallazgos así como los de otros grupos europeos, plantean una interrogante acerca de la real importancia de este polimorfismo en las diferentes poblaciones del mundo.

\section{CONCLUSIÓN}

Nuestros datos, obtenidos de una población chilena de CMT esporádico y controles, no apoyan la hipótesis de que la presencia de los polimorfismos G691S, L769L y S834S tenga importancia en la etiopatogenia del CMT, particularmente en la población chilena.

2. Wohшк N, Cote GJ, Evans DB, Goepfert H, Ordonez NG, GAGEL RF. Application of genetic screening information to the management of medullary thyroid carcinoma and multiple endocrine neoplasia type 2. Endocr Metab Clin North Am 1996; 25: 1-25. 
3. Mumgan LM, Kwok JBL, Healey C, Elsdon MJ, Eng C, GARDNER E ET AL. Germline mutations of the RET proto-oncogene in multiple endocrine neoplasia type 2A. Nature 1993; 363: 458-60.

4. Donis-Kelier H, Shenshen D, Chi D, Carlson KM, Toshima K, Lairmore TC et al. Mutations in the Ret proto-oncogene are associated with MEN 2A and FMTC. Hum Mol Genet 1993; 2: 851-6.

5. ENG C. RET proto-oncogene in the development of human cancer. J Clin Oncol 1999; 17: 380-93.

6. Gimm O, Neurberg DS, Marsh DJ, Dahia PL, HoangVu C, Raue F et aL. Over-representation of a germline RET sequence variant in patients with sporadic medullary thyroid carcinoma and somatic RET codon 918 mutation. Oncogene 1999; 18: 1369-73.

7. Ruiz A, Antinolo G, Fernández RM, Eng C, Marcos I, BORREGo S. Germline sequence variant S836S in the RET proto-oncogene is associated with low level predisposition to sporadic medullary thyroid carcinoma in the Spanish population. Clin Endocrinol (Oxf) 2001; 55: 399-402.

8. Elisei R, Cosci B, Romei C, Bottici V, Scuw M, Lari $\mathrm{R}$ ET AL. RET exon 11 (G691S) polymorphism is significantly more frequent in sporadic medullary thyroid carcinoma than in the general population. J Clin Endocrinol Metab 2004; 89: 3579-84.

9. Wiench M, Wygoda Z, Gubala E, Wloch J, LisowsKa K, KRASSOWSKI J ET AL. Estimation of risk of inherited medullary thyroid carcinoma in apparent sporadic patients. J Clin Oncol 2001; 19: 1374-80.

10. Leviev I, Negro F, James RW. Two alleles of the human paraoxonase gene produce different amounts of mRNA. An explanation for differences in serum concentrations of paraoxonase associated with the (Leu-Met54) polymorphism. Arterioscler Thromb Vasc Biol 1997; 17: 2935-9.

11. Berard I, Kraimps JL, Savagner F, Murat A, Renaudin K, NICOL-SIRE P ET AL Germline-sequence variants S836S and L769L in the RE arranged during Transfection (RET) proto-oncogene are not associated with predisposition to sporadic medullary carcinoma in the French population. Clin Genet 2004; 65: 150-2.

12. Wiench M, Wloch J, Wygoda Z, Gubala E, Oczko M, PAWLACZeK A ET AL. RET polymorphisms in codons 769 and 836 are not associated with predisposition to medullary thyroid carcinoma. Cancer Detect Prev 2004; 28: 231-6.
13. Wohuk N, Becker P, Youlton R, Cote GJ, Gagel RF. Mutaciones de línea germinal del proto-oncogen RET en pacientes chilenos con cáncer medular tiroideo familiar y esporádico. Rev Méd Chile 2001; 129: 713-8.

14. LAHIRI D, NuRNBERger J. A rapid non enzimatic method for the preparation of HMW DNA from blood for RFLP studies. J Nucleic Acid Res 1991; 19: 5444.

15. Berndt I, Reuter M, Saluer B, Frank-Raue K, Groth P, GRUBENDORF M ET AL. A new hot spot for mutations in the ret proto-oncogene causing familial medullary thyroid carcinoma and multiple endocrine neoplasia type 2A. J Clin Endocrinol Metab 1998; 83: 770-4.

16. Khorana S, Gagel $R$, Cote GJ. Direct sequencing of PCR products in agarose gel slices. Nucleic Acids Res 1994; 22: 3425-6.

17. Hofstra RM, LANDvaster RM, Ceccherini I, Stulp RP, Stelwagen T, Luo Y et al. A mutation in the RET proto-oncogene associated with multiple endocrine neoplasia type $2 \mathrm{~B}$ and sporadic medullary thyroid carcinoma. Nature 1994; 367: 375-6.

18. Eng C, Mumgan LM, Smith DP, Healey CS, Friung A, Raue F et aL. Mutation of the RET protooncogene in sporadic medullary thyroid carcinoma. Genes Chromosomes Cancer 1995; 12: 209-12.

19. Wоншк N, Cote GJ, Bugalho MMJ, Ordonez N, Evans DG, Goepfert H ET aL. Relevance of RET proto-oncogen mutations in sporadic medullary thyroid carcinoma. J Clin Endocrinol Metab 1996; 81: 3740-5.

20. Magalhães PK, De Castro M, Eluas LL, Soares SG, Maciel LM. Polymorphisms in the RET ProtoOncogene and the Phenotypic Presentation of Familial Medullary Thyroid Carcinoma. Thyroid 2004; 14: 848-52.

21. Cruz-Coke R, Moreno RS. Genetic epidemiology of single gene defects in Chile. J Med Genet 1994; 31: 702-6.

22. Wohwk N, Zapata R, Acuna M, Reyes H, Navarro A, RoA I ET AL. HFE gene mutations in Chile. Ann Intern Med 2003; 139: 708-9.

Agradecimientos

A los Drs. Robert F. Gagel y Gilbert J. Cote, por la enseñanza en las técnicas genéticas del proto-oncogen RET. A los socios de la Sociedad Chilena de Endocrinología y Metabolismo por permitimos estudiar sus pacientes. 